\title{
Haemophilus Influenzae B Vaccine
}

National Cancer Institute

\section{Source}

National Cancer Institute. Haemophilus Influenzae B Vaccine. NCI Thesaurus. Code

C1126.

A vaccine against serotype $b$ of the Gram-negative bacterium $\mathrm{H}$. influenzae (Hib). Hib vaccines contain polysaccharide-protein conjug ate antigens that produce greater host immune responses than first-generation purified polysaccharide vaccine. These vaccines may differ with regard to use of protein carriers, polysaccharides, diluents, and preservatives. 